\title{
Publisher Correction: Accelerated RNA detection using tandem CRISPR nucleases
}

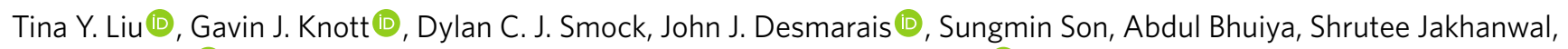
Noam Prywes (1), Shreeya Agrawal, María Díaz de León Derby, Neil A. Switz @i , Maxim Armstrong, Andrew R. Harris, Emeric J. Charles, Brittney W. Thornton, Parinaz Fozouni (1), Jeffrey Shu, Stephanie I. Stephens (1D, G. Renuka Kumar, Chunyu Zhao, Amanda Mok (D), Anthony T. lavarone, Arturo M. Escajeda (D), Roger Mclntosh, Shineui Kim, Eli J. Dugan (D), IGI Testing Consortium ${ }^{\star}$, Katherine S. Pollard (D), Ming X. Tan, Melanie Ott (D), Daniel A. Fletcher (D), Liana F. Lareau (D, Patrick D. Hsu (D), David F. Savage (1D) and Jennifer A. Doudna (D)

Correction to: Nature Chemical Biology https://doi.org/10.1038/s41589-021-00842-2, published online 5 August 2021.

In the version of this Article initially published, there were errors in the author affiliations, Fig. 3, main text and Acknowledgements section.

Specifically, the affiliation for author Ming X. Tan was mistakenly listed as affiliation 13, Center for Computational Biology, University of California, Berkeley, Berkeley, CA, USA. The correct affiliation for Ming X. Tan is with affiliation 15, Wainamics Inc., Pleasanton, CA, USA.

In Fig. 3d, a spurious black color key appeared above the red dotted line for detection accuracy; this has now been removed.

In the Results section, second paragraph, eighth sentence under heading "Detecting viral RNA with FIND-IT on a compact detector," the sentence now reading "Based on the $C_{t}$ values of all COVID-19-positive samples $(n=39)$ identified in samples from the IGI testing laboratory $(n=308)$," shows the correct number of samples from the IGI testing laboratory (308 vs. "296" as previously written).

In the Acknowledgements section, grant R33AI140465 was missing and has been added to the sentence now reading "the work was also supported by the Howard Hughes Medical Institute (HHMI) and the National Institutes of Health (NIH) (R01GM131073 and DP5OD021369 to P.D.H., R01GM127463 to D.F.S., and 5R61AI140465-03 and R33AI140465 to J.A.D., D.A.F. and M.O.).” Further in the Acknowledgements section, the following sentence has now been included: "The computational aspects of this work related to SARS-CoV-2 guide RNA design were supported by the AWS Diagnostic Development Initiative via computational credit."

The online version of the Article has been updated.

${ }^{\star} \mathrm{A}$ list of authors and their affiliations appears online.

Published online: 6 October 2021

https://doi.org/10.1038/s41589-021-00882-8

(c) The Author(s), under exclusive licence to Springer Nature America, Inc. 2021 